\title{
Initial stress formulation for three-dimensional elastoplastic analysis by the triple-reciprocity boundary element method
}

\author{
Y. Ochiai \\ Department of Mechanical Engineering, Kinki University, Japan
}

\begin{abstract}
In general, internal cells are required to solve elastoplastic problems using a conventional boundary element method (BEM). However, in this case, the merit of BEM, which is ease of data preparation, is lost. Triple-reciprocity BEM can be used to solve two-dimensional elastoplasticity problems with a small plastic deformation. It has been shown that three-dimensional elastoplastic problems can be solved, without the use of internal cells, by the triple-reciprocity BEM and initial strain method. In this study, an initial stress formulation is adopted and the initial stress distribution is interpolated using boundary integral equations. A new computer program was developed and applied to solving several problems.
\end{abstract}

Keywords: elastoplastic problem, initial stress method, BEM.

\section{Introduction}

Elastoplastic problems can be solved by a conventional boundary element method (BEM) using internal cells for domain integrals [1, 2]. In this case, however, the merit of BEM, which is ease of data preparation, is lost. On the other hand, several countermeasures have been considered. Ochiai and Kobayashi proposed the triple-reciprocity BEM (improved multiple-reciprocity BEM) without the use of internal cells for two-dimensional elastoplastic problems using an initial stress and strain formulations [3]. By this method, a highly accurate solution can be obtained using only fundamental solutions of a low order. It has been shown by Ochiai that three-dimensional elastoplastic problems can be solved, without the use of internal cells, by the triple-reciprocity BEM and initial strain method. 
In this study, the initial stress formulation and triple-reciprocity BEM are adapted to three-dimensional elastoplastic problems, and new fundamental solutions for this method are shown. In this method, only boundary elements and internal points are used. The arbitrary distributions of the initial stress for elastoplastic analysis are interpolated using boundary integral equations and internal points. In this method, strong singularities in the calculation of stresses at internal points become weak. A new computer program was developed and applied to several elastoplastic problems to clearly understand the theory.

\section{Theory}

\subsection{Initial stress formulation}

To analyze the elastoplastic problems using the initial strain formulation, the following boundary integral equation must be solved $[1,2]$.

$$
c_{i j}(P) \dot{u}_{j}(P)=\int_{\Gamma}\left[u_{i j}^{[1]}(P, Q) \dot{p}_{j}(Q)-p_{i j}(P, Q) \dot{u}_{j}(Q)\right] d \Gamma+\int_{\Omega} \varepsilon_{j k i}^{[1]}(P, q) \dot{\sigma}_{I j k}^{[1]}(q) d \Omega
$$

Here, $\dot{\sigma}_{I j k}^{[1]}$ is the initial stress rate and $c_{i j}$ is the free coefficient. Moreover, $\dot{u}_{i}$ and $\dot{p}_{i}$ are the $j$-th components of the displacement rate and the surface traction rate, respectively. On the other hand, $\Gamma$ and $\Omega$ are the boundary and the domain, respectively. As shown in Eq. (1), when there is an arbitrary initial stress rate, a domain integral becomes necessary. Denoting the distance between the observation point and the loading point by $r$, Kelvin's solution $u_{i j}^{[1]}$ and $p_{i j}$ are given by

$$
\begin{gathered}
u_{i j}^{[1]}=\frac{1}{16 \pi(1-v) G r}\left\{(3-4 v) \delta_{i j}+r,{ }_{i} r, j\right\} \\
p_{i j}=\frac{1}{8 \pi(1-v) G r^{2}}\left\{\left[(1-2 v) \delta_{i j}+3 r,{ }_{i} r,{ }_{j}\right] \frac{\partial r}{\partial n}-(1-2 v)\left(r,{ }_{i} n_{j}-r,{ }_{j} n_{i}\right)\right\},
\end{gathered}
$$

where $v$ is Poisson's ratio and $G$ is the shear modulus. The $i$-th component of a unit normal vector is denoted by $n_{i}$. Moreover, let us set $r,{ }_{i}=\partial r / \partial x_{i}$. The function $\varepsilon_{i j k}^{[1]}$ in Eq. (1) is given by [1]

$$
\varepsilon^{[1]}{ }_{j k i}=\frac{-1}{16 \pi(1-v) G r^{2}}\left\{(1-2 v)\left(\delta_{j i} r,_{k}+\delta_{k i} r,{ }_{j}\right)-\delta_{j k} r,{ }_{i}+3 r_{i} r,{ }_{j} r,{ }_{k}\right\} .
$$

\subsection{Interpolation of initial stress}

Interpolation using boundary integrals is introduced to avoid the domain integral in Eq. (1). The distribution of the initial stress $\dot{\sigma}_{I j k}^{[1]}$ in the case of a threedimensional problem is interpolated using the integral equation to transform the 
domain integral into a boundary integral. The following equations are used for interpolation [5-8]:

$$
\begin{gathered}
\nabla^{2} \dot{\sigma}_{I j k}^{[1] S}=-\dot{\sigma}_{I j k}^{[2] S}, \\
\nabla^{2} \dot{\sigma}_{I j k}^{[2] S}=-\sum_{m=1}^{M} \dot{\sigma}_{I j k(m)}^{[3] P A},
\end{gathered}
$$

where $\nabla^{2}=\partial^{2} / \partial x^{2}+\partial^{2} / \partial y^{2}+\partial^{2} / \partial z^{2}$. From Eqs. (6) and (7), we obtain

$$
\nabla^{4} \dot{\sigma}_{I j k}^{[1] S}=\sum_{m=1}^{M} \dot{\sigma}_{I j k(m)}^{[3] P A},
$$

where the function $\dot{\sigma}_{I j k}^{[3] P A}$ expresses a state of a uniformly distributed polyharmonic function in a spherical region with radius $A$. We must emphasize that Eqs. (6) and (7) can be used for interpolating the complicated distribution of the initial stress $\dot{\sigma}_{I j k}^{[1]}$. These equations are the same as those used to generate a free-form surface using an integral equation [6]. In this method, each component of initial stress $\dot{\sigma}_{I j k}^{[1]}(j, k=1,2,3)$ is interpolated.

\subsection{Representation of initial stress by integral equation}

The distribution of the initial stress is represented by an integral equation. The polyharmonic function $T^{[f]}$ and its normal derivatives are given by

$$
\begin{gathered}
T^{[f]}=\frac{r^{2 f-3}}{4 \pi(2 f-2) !} \\
\frac{\partial T^{[f]}}{\partial n}=\frac{(2 f-3) r^{2 f-4}}{4 \pi(2 f-2) !} \frac{\partial r}{\partial n}
\end{gathered}
$$

Figure 1 shows the shape of polyharmonic functions; the biharmonic function $T^{[2]}$ is not smooth at $r=0$. In the three-dimensional case, a smooth interpolation cannot be obtained using solely the biharmonic function $T^{[2]}$. In order to obtain a smooth interpolation, the polyharmonic function with volume distribution $T^{[2] A}$ is introduced. A polyharmonic function with volume distribution $T^{[f] A}$, as shown in Fig. 1, is defined as [5]

$$
T^{[f] A}=\int_{0}^{A}\left[\int_{0}^{2 \pi}\left\{\int_{0}^{\pi} T^{[f]} a^{2} \sin \theta d \theta\right\} d \phi\right] d a .
$$

The function $T^{[f] A}$ can be easily obtained using the relationships $r^{2}=R^{2}+a^{2}-2 a R \cos \theta$ and $d r=a R \sin \theta d \theta$, as shown in Fig.1. This function is written using $r$ instead of $R$, similarly to Eqs. (8) and (9), though the function in Eq. (10) is a function of $R$. The newly defined function $T^{[f] A}$ can be explicitly shown as

$$
T^{[f] A}=\frac{1}{2 r(2 f+1) !}\left\{(2 f A-r)(r+A)^{2 f}+(2 f A+r)(r-A)^{2 f}\right\} \quad r>A
$$




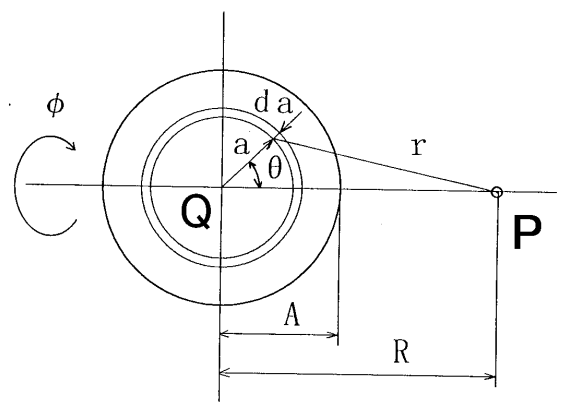

Figure 1: Notations for polyharmonic function with volume distribution.

$$
T^{[f] A}=\frac{1}{2 r(2 f+1) !}\left\{(2 f A-r)(A+r)^{2 f}-(2 f A+r)(A-r)^{2 f}\right\} \quad r \leq A .
$$

Denoting the number of points $\dot{\sigma}_{I j k}^{[3] P}$ as $M$, the curvature of the initial stress rate $\dot{\sigma}_{I j k}^{[2] S}$ is given by Green's second identity and Eq. (6) as [4-6]

$$
\begin{gathered}
c \dot{\sigma}_{I j k}^{[2] S}(P)=\int_{\Gamma}\left\{T^{[1]}(P, Q) \frac{\partial \dot{\sigma}_{I j k}^{[2] S}(Q)}{\partial n}-\frac{\partial T^{[1]}(P, Q)}{\partial n} \dot{\sigma}_{I j k}^{[2] S}(Q)\right\} d \Gamma \\
+\sum_{m=1}^{M} T^{[1] A}(P, q) \dot{\sigma}_{I j k(m)}^{[3] P A}(q) \cdot
\end{gathered}
$$

The initial stress rate $\dot{\sigma}_{I j k}^{[1]}$ is given by Green's theorem and Eqs. (5) and (6) as [4-6]

$$
\begin{gathered}
c \dot{\sigma}_{I j k}^{[1] S}(P)=-\sum_{f=1}^{2}(-1)^{f} \int_{\Gamma}\left\{T^{[f]}(P, Q) \frac{\partial \dot{\sigma}_{I j k}^{[f] S}(Q)}{\partial n}-\frac{\partial T^{[f]}(P, Q)}{\partial n} \dot{\sigma}_{I j k}^{[f] S}(Q)\right\} d \Gamma \\
-\sum_{m=1}^{M} T^{[2] A}(P, q) \dot{\sigma}_{I j k(m)}^{[3] P A}(q),
\end{gathered}
$$

where $\mathrm{c}=0.5$ on the smooth boundary and $c=1$ in the domain. It is assumed that $\dot{\sigma}_{I j k}^{[2] S}(\mathrm{Q})$ is zero. For internal points, the next equation is obtained similarly to Eq. (14).

$$
\begin{gathered}
c \dot{\varepsilon}_{I j k}^{[1] S}(p)=-\sum_{f=1}^{2}(-1)^{f} \int_{\Gamma}\left\{T^{[f]}(p, Q) \frac{\partial \dot{\varepsilon}_{I j k}^{[f] S}(Q)}{\partial n}-\frac{\partial T^{[f]}(p, Q)}{\partial n} \dot{\varepsilon}_{I j k}^{[f] S}(Q)\right\} d \Gamma \\
-\sum_{m=1}^{M} T^{[2] A}(p, q) \dot{\varepsilon}_{I j k(m)}^{[3] P A}(q)
\end{gathered}
$$

If the boundary is divided into $N_{0}$ constant elements, and $N_{1}$ internal points are used, the simultaneous linear algebraic equations with $\left(2 N_{0}+N_{l}\right)$ as unknowns must be solved. 


\subsection{Triple-reciprocity boundary element method for the representation of initial stress}

The function $\varepsilon_{j k i}^{[f]}$ is defined as

$$
\nabla^{2} \varepsilon_{j k i}^{[f+1]}=\varepsilon_{j k i}^{[f]} .
$$

Using eqs (5), (6) and (16) and Green's second identity, eq (1) becomes

$$
\begin{aligned}
c_{i j}(P) \dot{u}_{j}(P) & =\int_{\Gamma}\left[u_{i j}^{[1]}(P, Q) \dot{p}_{j}(Q)-p_{i j}(P, Q) \dot{u}_{j}(Q)\right] d \Gamma-\sum_{f=1}^{2}(-1)^{f} \int_{\Gamma}\left\{\frac{\partial \varepsilon_{j k i}^{[f+1]}(P, Q)}{\partial n} \dot{\sigma}_{I j k}^{[f] S}(Q)\right. \\
& \left.-\varepsilon_{j k i}^{[f+1]}(P, Q) \frac{\partial \dot{\sigma}_{I j k}^{[f] S}(Q)}{\partial n}\right\} d \Gamma+\sum_{m=1}^{M} \varepsilon_{j k i}^{[3] A}(P, q) \dot{\sigma}_{I}^{[j] k(m)}(q)
\end{aligned}
$$

$\varepsilon_{i j k}^{[f]}$ for the representation of initial stress is obtained as [4]

$$
\begin{aligned}
& \varepsilon_{i j k}^{[f]}=\frac{(2 f-1)(2 f-3) r^{2 f-4}}{8 \pi(1-v)(2 f) ! G} \\
& \left\{(2 f-1-2 f v)\left(\delta_{j k} r,{ }_{i}+\delta_{i k} r,{ }_{j}\right)-\delta_{i j} r,{ }_{k}-(2 f-5) r,{ }_{i} r,{ }_{j} r,{ }_{k}\right\} .
\end{aligned}
$$

Moreover, the normal derivative $\partial \varepsilon_{i j k}^{[f]} / \partial n$ and $\varepsilon_{i j k}^{[3] A}$ are given by

$$
\begin{aligned}
& \frac{\partial \varepsilon^{[f]}{ }_{i j k}}{\partial n}=\frac{(2 f-1)(2 f-3) r^{2 f-5}}{8 \pi(1-v)(2 f) ! G}\left[( 2 f - 5 ) \left\{(2 f-1-2 f v)\left(\delta_{j k} r,_{i}+\delta_{i k} r,_{j}\right)\right.\right. \\
& \left.-\delta_{i j} r,{ }_{k}-(2 f-7) r,{ }_{i} r,{ }_{j} r,{ }_{k}\right\} \frac{\partial r}{\partial n}-(2 f-5)\left(r,{ }_{j} r,_{k} n_{i}+r,{ }_{i} r{ }_{k} n_{j}+r,{ }_{i} r,{ }_{j} n_{k}\right) \\
& \left.+(2 f-1-2 f v)\left(\delta_{j k} n_{i}+\delta_{i k} n_{j}\right)-\delta_{i j} n_{k}\right] \\
& \varepsilon_{i j k}^{[3] A}=\frac{A^{3}}{15120(1-v) r^{4} G}\left\{-\left(\delta_{j k} r,_{i}+\delta_{i k} r,{ }_{j}+\delta_{i j} r,_{k}\right)\left(105 r^{6}+63 A^{2} r^{4}-9 A^{4} r^{2}+A^{6}\right)\right. \\
& -r,{ }_{i} r,{ }_{j} r{ }_{k}\left(105 r^{6}-63 A^{2} r^{4}+27 A^{4} r^{2}-5 A^{6}\right) \\
& \left.+18(1-v)\left(\delta_{j k} r,_{i}+\delta_{i k} r,{ }_{j}\right) r^{2}\left(35 r^{4}+14 A^{2} r^{2}-A^{4}\right)\right\} \quad r>A \\
& \varepsilon_{i j k}^{[3] A}=\frac{r}{7560(1-v) G}\left\{-\left(\delta_{j k} r,_{i}+\delta_{i k} r,{ }_{j}+\delta_{i j} r,{ }_{k}\right)\left(-r^{4}+18 A^{2} r^{2}+63 A^{4}\right)\right. \\
& \left.-4 r,{ }_{i} r,{ }_{j} r,{ }_{k} r^{2}\left(-r^{2}+9 A^{2}\right)+9(1-v)\left(\delta_{j k} r{ }_{i}+\delta_{i k} r,{ }_{j}\right)\left(-r^{4}+14 A^{2} r^{2}+35 A^{4}\right)\right\}
\end{aligned}
$$

\subsection{Internal stresses}

The internal stress is given by

$$
\begin{gathered}
\dot{\sigma}_{i j}(p)=\int_{\Gamma}\left[-\sigma_{k i j}^{[1]}(p, Q) \dot{p}_{k}(Q)-S_{k i j}(p, Q) \dot{u}_{k}(Q)\right] d \Gamma \\
+\int_{\Omega} \varepsilon_{i j k l}^{[1]}(p, q) \dot{\sigma}_{I k l}^{[1]}(q) d \Omega-\dot{\sigma}_{I i j}^{[1]}(q),
\end{gathered}
$$


where $\dot{\sigma}_{I i j}^{[1]}$ is the initial stress obtained. The functions $S_{k i \mathrm{j}}$ and $\sigma_{i j k s}^{[1]}$ in Eq. (22) are given by

$$
\begin{aligned}
& S_{k i j}=\frac{G}{2 \pi(1-v) r^{2}}\left[2 \frac{\partial r}{\partial n}\left\{(1-2 v) \delta_{i j} r_{, k}+v\left(\delta_{i k} r_{, j}+\delta_{j k} r_{, i}\right)-4 r_{, j} r_{, j} r_{, k}\right\}\right. \\
&\left.+2 v\left(n_{i} r_{, j} r_{, k}+n_{j} r_{, i} r_{, k}\right)+(1-2 v)\left(2 n_{k} r_{, i} r_{, j}+n_{j} \delta_{i k}+n_{i} \delta_{j k}\right)-(1-4 v) n_{k} \delta_{i j}\right] \\
& \sigma_{i j k l}^{[1]}= \frac{1}{2 \pi(1-v) r^{2}}\left[2(1-2 v)\left(\delta_{i j} r_{, k} r_{, l}+\delta_{k l} r_{, i} r_{, j}\right)\right. \\
&+2 v\left(\delta_{i l} r_{, j} r_{, k}+\delta_{j k} r_{, i} r_{l}+\delta_{i k} r_{, j} r_{, s}+\delta_{j l} r_{, i} r_{, k}\right) \\
&\left.+(1-2 v)\left(\delta_{i k} \delta_{l j}+\delta_{j k} \delta_{l i}\right)-(1-4 v) \delta_{i j} \delta_{k l}-8 r_{, i} r_{, j} r_{, k} r_{l}\right] .
\end{aligned}
$$

The function $\varepsilon_{i j k l}^{[f]}$ is defined as

$$
\nabla^{2} \varepsilon_{i j k l}^{[f+1]}=\varepsilon_{i j k l}^{[f]} .
$$

Using Eq. (25) and Green's theory, Eq. (22) becomes

$$
\begin{gathered}
\dot{\sigma}_{i j}(p)=\int_{\Gamma}\left[-\sigma_{k i j}^{[1]}(p, Q) \dot{p}_{k}(Q)-S_{k i j}(p, Q) \dot{u}_{k}(Q)\right] d \Gamma \\
-\sum_{f=1}^{2}(-1)^{f} \int_{\Gamma}\left[\frac{\partial \sigma_{i j k l}^{[f+1]}(p, Q)}{\partial n} \dot{\varepsilon}_{I k l}^{[f] S}(Q)-\sigma_{i j k l}^{[f+1]}(p, Q) \frac{\partial \dot{\varepsilon}_{I k l}^{[f]}(Q)}{\partial n}\right] d \Gamma \\
+\sum_{m=1}^{M} \varepsilon_{i j k l}^{[3] A}(p, q) \dot{\sigma}_{I k l(m)}^{[3] P A}(q)-\dot{\sigma}_{I i j}^{[1]}(q)
\end{gathered}
$$

Using Eq. (22) and the relationship between displacement and strain, $\varepsilon_{i j k l}^{[f]}$ is obtained as

$$
\begin{aligned}
& \varepsilon_{i j k l}^{[f]}=\frac{(2 f-1)(2 f-3) r^{2 f-5}}{4 \pi(1-v)(2 f) !}\left\langle\left[(2 f-1-2 f v)\left(\delta_{i k} \delta_{j l}+\delta_{i l} \delta_{j k}\right)\right.\right. \\
& +(2 f-5)(f-1-f v)\left(\delta_{j l} r{ }_{i} r,{ }_{k}+\delta_{j k} r,{ }_{i} r,{ }_{l}+\delta_{i l} r,{ }_{j} r,{ }_{k}+\delta_{i k} r,{ }_{j} r,{ }_{l}\right) \\
& -(1-2 f v)\left\{(2 f-5) \delta_{k l} r,{ }_{i} r,{ }_{j}+\delta_{i j} \delta_{k l}\right\}+(2 f-5)+\delta_{i j} r,{ }_{k} r,{ }_{l} \\
& \left.-(2 f-5)(2 f-7) r,{ }_{i} r,{ }_{j} r,{ }_{k} r, l\right]>\text {. }
\end{aligned}
$$

Similarly, $\partial \varepsilon_{i j k l}^{[f]} / \partial n$ and $\varepsilon_{i j k l}^{[3] A}$ are obtained as

$$
\begin{aligned}
& \frac{\partial \varepsilon^{[f]}{ }_{i j k l}}{\partial n}=\frac{(2 f-1)(2 f-3)(2 f-5) r^{2 f-6}}{2 \pi(1-v)(2 f) !}\left\langle\left[(2 f-1-2 f v)\left(\delta_{i k} \delta_{j l}+\delta_{i l} \delta_{j k}\right)\right.\right.
\end{aligned}
$$

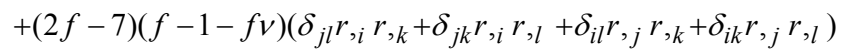

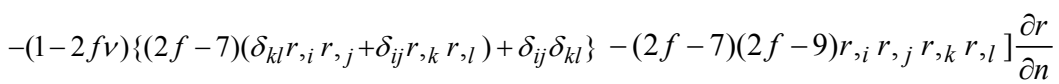

$$
\begin{aligned}
& +(f-1-f v)\left\{\left(\delta_{j l} n_{k}+\delta_{j k} n_{l}\right) r,_{i}+\left(\delta_{i l} n_{k}+\delta_{i k} n_{l}\right) r,{ }_{j}\right. \\
& \left.+\left(\delta_{j l} n_{i}+\delta_{i l} n_{j}\right) r,_{k}+\left(\delta_{j k} n_{i}+\delta_{i k} n_{j}\right) r, l\right\}
\end{aligned}
$$




$$
\begin{aligned}
& -(1-2 f v)\left\{\delta_{i j}\left(r, n_{l} n_{k}+r,{ }_{k} n_{l}\right)+\delta_{k l}\left(r,{ }_{j} n_{i}+r,{ }_{i} n_{j}\right)\right\} \\
& \left.-(2 f-7)\left\{\left(r,{ }_{l} n_{k}+r,{ }_{k} n_{l}\right) r,{ }_{i} r,{ }_{j}+\left(r,{ }_{j} n_{i}+r_{, i} n_{j}\right) r,{ }_{k} r, l\right\}\right\rangle \\
& \varepsilon_{i j k l}^{[3] A}=\frac{A^{3}}{7560(1-v) r^{5}}\left[1 8 v r ^ { 2 } \delta _ { i j } \left\{\delta_{k l}\left(35 r^{4}+14 A^{2} r^{2}-A^{4}\right)+r,{ }_{k} r, l\left(35 r^{4}-14 A^{2} r^{2}\right.\right.\right. \\
& \left.\left.+3 A^{4}\right)\right\}-\left(\delta_{i j} \delta_{k l}+\delta_{k j} \delta_{l i}+\delta_{k i} \delta_{l i}\right)\left(105 r^{6}+63 A^{2} r^{4}-9 A^{4} r^{2}+A^{6}\right)
\end{aligned}
$$

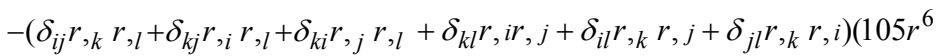

$$
\begin{aligned}
& \left.-63 A^{2} r^{4}+27 A^{4} r^{2}-5 A^{6}\right)-r,{ }_{j} r{ }_{i} r,{ }_{k} r, l\left(-105 r^{6}+189 A^{2} r^{4}-135 A^{4} r^{2}+35 A^{6}\right) \\
& +9(1-v) r^{2}\left\{2\left(\delta_{k i} \delta_{j l}+\delta_{k j} \delta_{i l}\right)\left(35 r^{4}+14 A^{2} r^{2}-A^{4}\right)\right. \\
& \left.\left.+\left(\delta_{k i} r,{ }_{j} r_{l}+\delta_{k j} r{ }_{i} r_{l}+\delta_{l i} r,{ }_{j} r,{ }_{k}+\delta_{l j} r{ }_{i} r{ }_{k}\right)\left(35 r^{4}-14 A^{2} r^{2}+3 A^{4}\right)\right\}\right] \quad r>A \text { (29) } \\
& \varepsilon_{i j k l}^{[3] A}=\frac{1}{3780(1-v)}\left[9 v \delta_{i j}\left\{\delta_{k l}\left(-r^{4}+14 A^{2} r^{2}+35 A^{4}\right)+4 r,{ }_{k} r,{ }_{, l} r^{2}\left(-r^{2}+7 A^{2}\right)\right\}\right. \\
& -\left(\delta_{i j} \delta_{k l}+\delta_{i k} \delta_{j l}+\delta_{i l} \delta_{j k}\right)\left(-r^{4}+18 A^{2} r^{2}+63 A^{4}\right)
\end{aligned}
$$

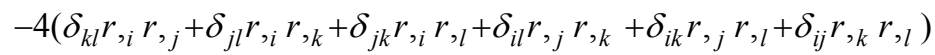

$$
\begin{aligned}
& r^{2}\left(-r^{2}+9 A^{2}\right)+8 r,{ }_{i} r,{ }_{j} r,{ }_{k} r,{ }_{l} r^{4}+9(1-v)\left\{\left(\delta_{i k} \delta_{j l}+\delta_{i l} \delta_{j k}\right)\left(-r^{4}+14 A^{2} r^{2}+35 A^{4}\right)\right.
\end{aligned}
$$

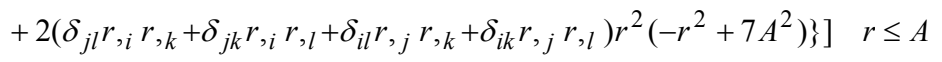

\section{Numerical examples}

In order to ensure the accuracy of the present method, the stress in a thick cylinder, which is made of an elastoplastic material, subjected to internal pressure is obtained. It is assumed that the inner and outer radii are 10 and $30 \mathrm{~mm}$. The von Mises yield criterion is used, and the cylinder is free in the $\mathrm{z}$ direction. Young's modulus $E=210 \mathrm{GPa}$ and Poisson's ratio $v=0.30$ are assumed. Internal pressure $p_{O}=1.2 \mathrm{GPa}$, yield stress $\sigma_{Y}=1.2 \mathrm{GPa}$ and strain hardening $H=0.1 E$ are assumed. The numbers of discretized boundary elements and internal points are 680 and 315, as shown in Fig. 2. Internal points are used to interpolate the distribution of initial stress. Figure 3 shows the circumferential and radial stress distributions. Boundary element results are shown with FEM solutions in Fig. 2. The stress distributions agree well with the FEM solutions.

Next elastoplastic problem is a notched tensile specimen which is shown in Fig.4. This example is one of the very early plasticity problems solved using the finite-element technique, and boundary element solutions were presented by Telles [1]. Von Mises yield criterion and no-strain hardening is assumed. The number of discretized boundary elements is 856 , and the number of internal points for interpolation is 715 , as shown in Fig. 5. Young's modulus $E=70.0 \mathrm{GPa}$, Poisson's ratio $\nu=0.2$ and yield stress $\sigma_{0}=243 \mathrm{MPa}$ are assumed. The spread of plastic zones at different load levels presented in Fig.6 exhibits good agreement with the conventional boundary element solution for the same problem. 


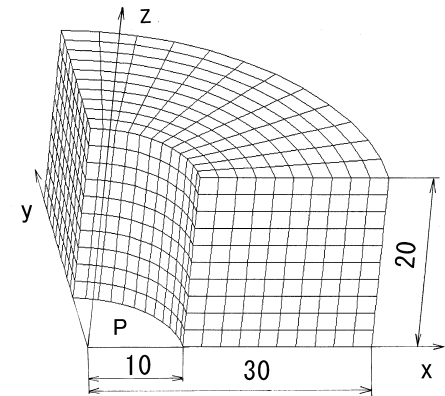

(a)

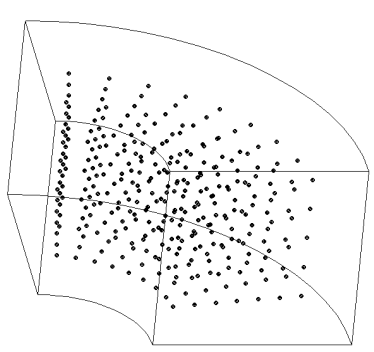

(b)

Figure 2: Boundary elements and internal points in quarter-region (Number of boundary elements: 680). (a) Boundary elements, (b) internal points.
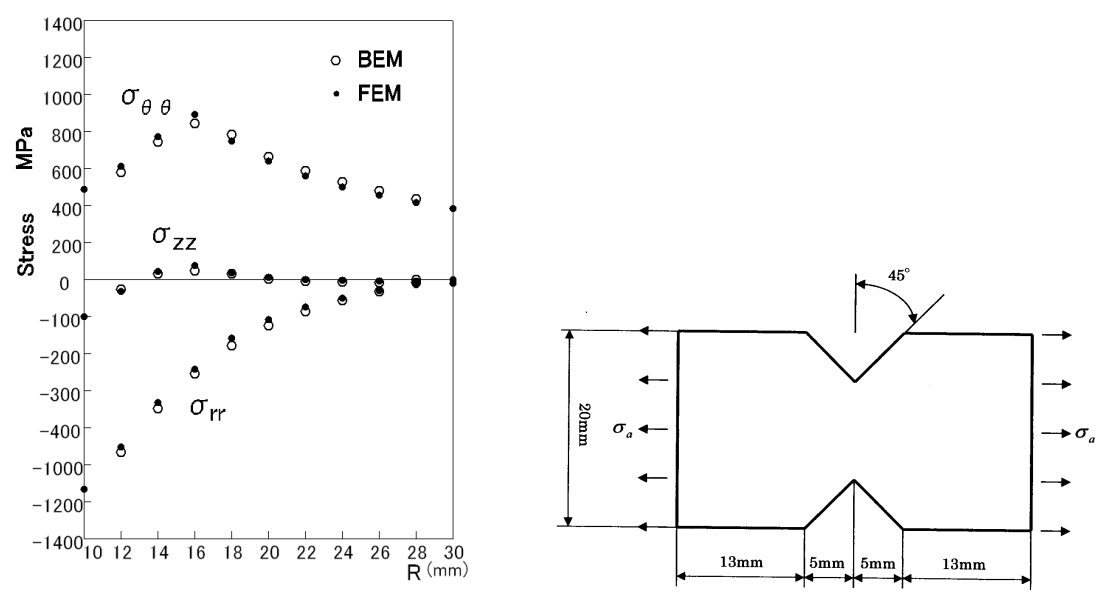

Figure 3: Stress distribution in hollow cylinder with internal pressure.

Figure 4: Notched tensile specimen.

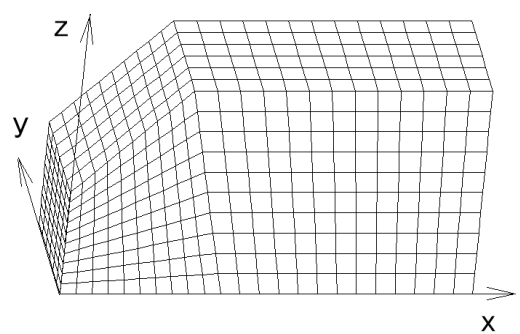

(a)

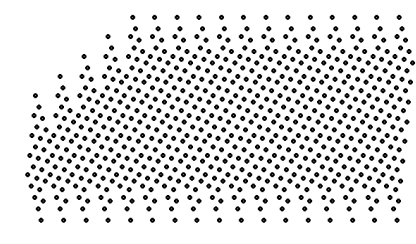

(b)

Figure 5: Notched tensile specimen. (a) Boundary elements, (b) internal points. 


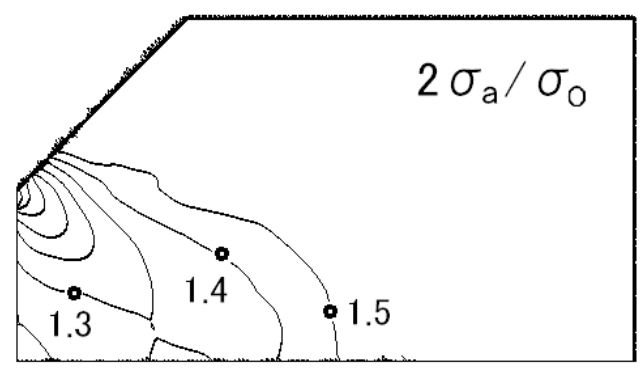

Figure 6: Plastic zone obtained for various values of $2 \sigma_{a} / \sigma_{0}$.

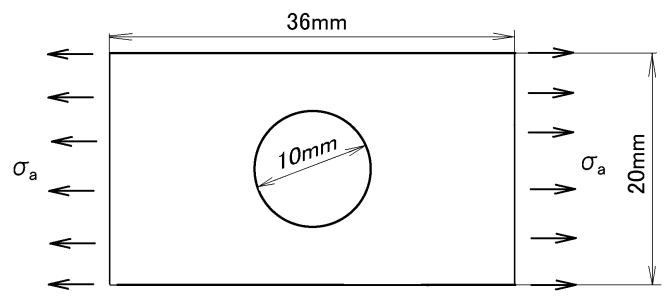

Figure 7: $\quad$ Perforated tension strip.

The elastoplastic problem of a plate with a circle hole, as shown in Fig. 7, is solved by using Mises yield criterion. Uniform traction is $\sigma_{\mathrm{a}}$, and the thickness of the plate is $1 \mathrm{~mm}$. The number of discretized boundary elements is 856 , and the number of internal points for interpolation is 660 as shown in Fig.8. Young's modulus $E=70 \mathrm{GPa}$, Poisson's ratio $v=0.2$ and the yield stress $\sigma_{\mathrm{Y}}=243 \mathrm{MPa}$ are assumed. Fig.9 shows the plastic zone obtained by this method for various values $2 \sigma_{\mathrm{a}} / \sigma_{\mathrm{Y}}$ in a quarter region. This result is in good agreement with the FEM analysis.

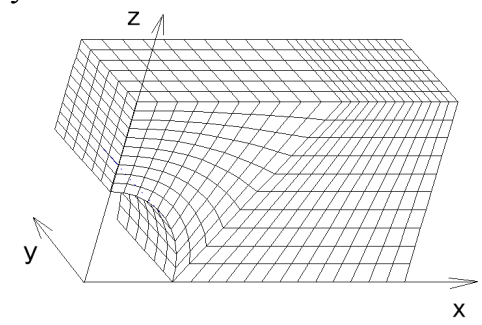

(a)

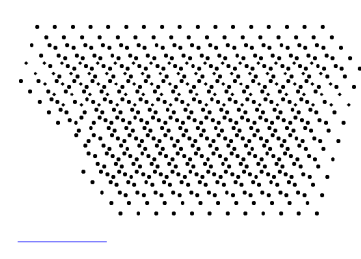

(b)

Figure 8: Boundary element in quarter region. (a) Boundary elements, (b) internal points. 


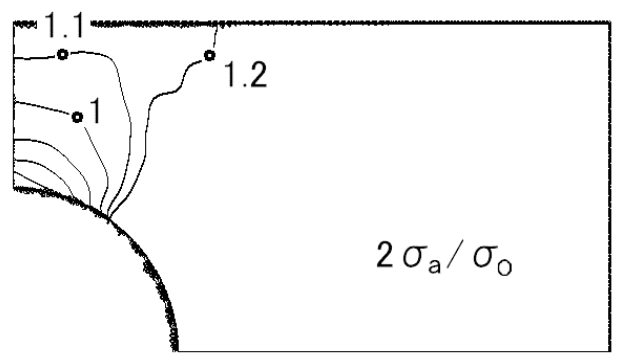

Figure 9: $\quad$ Plastic zone obtained for various values of $2 \sigma_{a} / \sigma_{0}$.

\section{Conclusion}

It was shown that three-dimensional elastoplastic analysis can be carried out, without the use of internal cells, using the triple-reciprocity boundary element method and initial stress method. The fundamental solutions for initial stress method were shown. In this method, the strong singularity that appears in the calculation of internal stress by the conventional boundary element method becomes weak. Using numerical examples, the effectiveness and accuracy of this method were demonstrated. In this method, the merit of BEM, which is ease of data preparation, is not lost because internal cells are not necessary.

\section{References}

[1] Telles, J. C. F., The Boundary Element Method Applied to Inelastic Problems, Springer-Verlag, Berlin, 1983.

[2] Ochiai, Y. and Kobayashi, T., Initial Stress Formulation for Elastoplastic Analysis by Improved Multiple-Reciprocity Boundary Element Method, Engineering Analysis with Boundary Elements, Vol. 23, pp. 167-173, 1999.

[3] Ochiai, Y. and Kobayashi, T., Initial Strain Formulation without Internal Cells for Elastoplastic Analysis by Triple-Reciprocity BEM, International Journal for Numerical Methods in Engineering, Vol. 50, pp. 1877-1892, 2001.

[4] Ochiai, Y. and Sladek, V. Numerical Treatment of Domain Integrals without Internal Cells in Three-Dimensional BIEM Formulations, CMES (Computer Modeling in Engineering \& Sciences), Vol. 6, No. 6, pp. 525-536, 2004.

[5] Ochiai, Y., Nishitani, H. and Sekiya, T., Stress Analysis with Arbitrary Body Force by Boundary Element Method, Engineering Analysis with Boundary Elements, Vol. 17, pp. 295-302, 1996.

[6] Ochiai, Y., Multidimensional Numerical Integration for Meshless BEM, Engineering Analysis with Boundary Elements, Vol. 27, No. 3, pp. 241-249, 2003. 